\title{
A matrix isolation and computational study of molecular palladium fluorides: does $\mathrm{PdF}_{6}$ exist?
}

Antony V. Wilson, ${ }^{\dagger, \# ~ T i m o t h y ~ N g u y e n, ~}{ }^{\ddagger}$ Felix Brosi, ${ }^{\S}$ Xuefeng Wang, ${ }^{\uparrow}, \|$ Lester Andrews,

Sebastian Riedel, ${ }^{\S, \perp}$ Adam J. Bridgeman, ${ }^{\ddagger}$ and Nigel A. Young* ${ }^{\star}$

${ }^{\dagger}$ Department of Chemistry, The University of Hull, Kingston upon Hull, HU6 7RX, U.K.

* School of Chemistry, The University of Sydney, NSW 2006, Australia

${ }^{\S}$ Institut für Chemie und Biochemie - Anorganische Chemie, Freie Universität Berlin, Fabeckstrasse 34-36, D-14195 Berlin, Germany

" Department of Chemistry, University of Virginia, Charlottesville, Virginia, VA, 22904-4319, USA

${ }^{\perp}$ Institut für Anorganische und Analytische Chemie, Albert-Ludwigs-Universität Freiburg,

Albertstrasse 21, D-79104 Freiburg im Breisgau, Germany

*n.a.young@hull.ac.uk

441482465442 (tel)

Current addresses

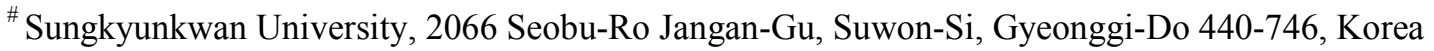

" Department of Chemistry, Tongji University, Shanghai, 200092, China. 


\section{Supplementary Material}

Table S1 Summary of computational results

\begin{tabular}{|c|c|c|c|c|c|c|}
\hline & & & $\mathrm{Pd}-\mathrm{F} / \AA$ & F-Pd-F $/{ }^{\circ}$ & $v_{\text {Pd-F }}$ modes $/ \mathrm{cm}^{-1}$ (symmetry species, IR intensity $\mathrm{km} \mathrm{mol}^{-1}$ ) & Ref \\
\hline \multirow[t]{5}{*}{$\overline{\mathrm{PdF}}$} & $C_{\infty v}$ & ${ }^{2} \Sigma^{+}$ & 1.930 & & $533.9\left(\Sigma^{+}, 41\right)$ & (a) \\
\hline & $C_{\infty v}$ & ${ }^{2} \Sigma^{+}$ & 1.931 & & $542.7\left(\Sigma^{+}, 60\right)$ & (b) \\
\hline & $C_{\infty v}$ & ${ }^{2} \Sigma^{+}$ & 1.99 & & & (d) \\
\hline & $C_{\infty v}$ & ${ }^{2} \Sigma^{+}$ & 2.01 & & & (e) \\
\hline & $C_{\infty}$ & ${ }^{2} \Sigma^{+}$ & 1.961 & & 531 & (f) \\
\hline \multirow[t]{3}{*}{$\overline{\mathrm{PdF}_{2}}$} & $D_{\infty h}$ & ${ }^{3} \Pi_{g}$ & 1.895 & 180 & $633.5\left(\Sigma_{\mathrm{u}}^{+}, 146\right), 560.9\left(\Sigma_{\mathrm{g}}^{+}, 0\right)$ & (a) \\
\hline & $D_{\infty h}$ & ${ }^{3} \Pi_{g}$ & 1.878 & 180 & $655.0\left(\Sigma_{\mathrm{u}}^{+}, 183\right), 573.2\left(\Sigma_{\mathrm{g}}^{+}, 0\right)$ & (b) \\
\hline & $D_{\infty h}$ & ${ }^{3} \Pi_{g}$ & 1.94 & 180 & & (g) \\
\hline$\overline{\mathrm{PdF}_{3}}$ & $C_{2 v}$ & ${ }^{2} \mathrm{~A}_{2}$ & $\begin{array}{l}1.865(\mathrm{x} 2) \\
1.886\end{array}$ & $\begin{array}{l}168.0 \\
96.0\end{array}$ & $661.8\left(\mathrm{~B}_{2}, 117\right), 591.9\left(\mathrm{~A}_{1}, 0.86\right), 565.3\left(\mathrm{~A}_{1}, 39.7\right)$ & (a) \\
\hline \multirow[t]{3}{*}{$\overline{\mathrm{PdF}_{4}}$} & $\begin{array}{l}D_{4 h} \\
D_{4 h}{ }^{\dagger} \\
D_{2 h}^{\dagger}\end{array}$ & $\begin{array}{l}\mathrm{A}_{1}\left({ }^{3} \mathrm{E}_{\mathrm{g}}\right) \\
{ }^{3} A_{1 g} \\
{ }^{3} B_{2 g}\end{array}$ & $\begin{array}{l}1.866 \\
1.850 \\
1.861(\mathrm{x} 2) \\
1.825(\mathrm{x} 2)\end{array}$ & $\begin{array}{l}90 \\
90 \\
90\end{array}$ & $\begin{array}{l}663.5\left(\mathrm{E}_{\mathrm{u}}, 81.6 \times 2\right), 585.6\left(\mathrm{~A}_{1 \mathrm{~g}}, 0\right), 523.7\left(\mathrm{~B}_{1 \mathrm{~g}}, 0\right) \\
696.5\left(\mathrm{E}_{\mathrm{u}}, 70 \times 2\right), 618.0\left(\mathrm{~A}_{1 \mathrm{~g}}, 0\right), 578.1\left(\mathrm{~B}_{1 \mathrm{~g}}, 0\right) \\
714.4\left(\mathrm{~B}_{2 \mathrm{u}}, 112\right), 681.9\left(B_{1 u}, 120\right), 627.1\left(A_{g}, 0\right), 561.9\left(A_{g}, 0\right)\end{array}$ & $\begin{array}{l}\text { (a) } \\
\text { (b) } \\
\text { (b) }\end{array}$ \\
\hline & $D_{2 h}$ & ${ }^{3} B_{2 g}$ & $\begin{array}{l}1.848(\mathrm{x} 2) \\
1.805(\mathrm{x} 2)\end{array}$ & 90 & $750.4\left(\mathrm{~B}_{2 \mathrm{u}}, 135\right), 707.7\left(B_{3 u}, 171\right), 644.6\left(A_{g}, 0\right), 578.2\left(A_{g}, 0\right)$ & (c) \\
\hline & $D_{2 d}$ & & $1.888(\mathrm{x} 4)$ & 167.4 & $592(E, 78), 558\left(A_{1}, 0\right), 504\left(B_{2}, 6\right)$ & (h) \\
\hline \multirow[t]{4}{*}{$\overline{\mathrm{PdF}_{5}}$} & $C_{4 v}$ & ${ }^{2} \mathrm{~B}_{2}$ & $\begin{array}{l}1.878(\mathrm{x} 4) \\
1.854\end{array}$ & $\begin{array}{l}173.03 \\
93.5,89.8\end{array}$ & $648.3(\mathrm{E}, 79.1 \times 2), 632.7\left(\mathrm{~A}_{1}, 15.5\right), 578.6\left(\mathrm{~A}_{1}, 0.95\right), 552.6\left(\mathrm{~B}_{1}, 0\right)$ & (a) \\
\hline & $C$ & ${ }^{2} \mathrm{~B}_{1}$ & $1.837(\mathrm{x} 1)$ & 89.9 & $687.6(\mathrm{E}, 128 \times 2), 674.3\left(\mathrm{~A}_{1}, 8\right), 621.1\left(\mathrm{~A}_{1}, 0\right), 576.7\left(\mathrm{~B}_{2}, 0\right)$ & (b) \\
\hline & $4 v$ & & $1.850(\mathrm{x} 4)$ & 92.7 & & \\
\hline & $C_{4 v}$ & ${ }^{2} \mathrm{~B}_{1}$ & $\begin{array}{l}1.836(\mathrm{x} 1) \\
1.836(\mathrm{x} 4)\end{array}$ & 92.1 & & (c) \\
\hline \multirow{12}{*}{$\overline{\mathrm{PdF}_{6}}$} & $O_{h}$ & $A_{1}\left({ }^{3} E_{g}\right)$ & 1.876 & 180,90 & $648.0\left(\mathrm{~T}_{1 \mathrm{u}}, 58.7 \times 3\right), 574.0\left(\mathrm{~A}_{1 \mathrm{~g}}, 0\right), 541.2\left(\mathrm{E}_{\mathrm{g}}, 0 \times 2\right)$ & (a) \\
\hline & $D_{4 h}$ & ${ }^{3} A_{\lg }$ & $\begin{array}{l}1.853 \\
1.839\end{array}$ & & $\begin{array}{l}694.7\left(\mathrm{~A}_{2 \mathrm{u}}, 124\right), 672.4\left(\mathrm{E}_{\mathrm{u}}, 41 \times 2\right), 611.8\left(\mathrm{~A}_{1 \mathrm{~g}}, 0\right), 580.5\left(\mathrm{~B}_{1 \mathrm{~g}}, 0\right) \\
570.9\left(\mathrm{~A}_{1 \mathrm{~g}}, 0\right)\end{array}$ & (b) \\
\hline & $D_{4 h}$ & ${ }^{3} A_{\lg }$ & $\begin{array}{l}1.841 \\
1.827\end{array}$ & & $\begin{array}{l}724.5\left(\mathrm{~A}_{2 \mathrm{u}}, 122\right), 718.0\left(\mathrm{E}_{\mathrm{u}}, 33 \mathrm{x} 2\right), 646.5\left(\mathrm{~A}_{1 \mathrm{~g}}, 0\right), 585.2\left(\mathrm{~B}_{2 \mathrm{~g}}, 0\right) \\
544.2\left(\mathrm{~A}_{1 \mathrm{~g}}, 0\right)\end{array}$ & (c) \\
\hline & $D_{2 d}$ & & $1.894(\mathrm{x} 4)$ & $\begin{array}{l}92.2 \\
87.8\end{array}$ & $585\left(B_{2}, 100\right), 544\left(A_{1}, 0\right), 530(E, 8), 505\left(A_{1}, 0\right), 503\left(B_{1}, 0\right)$ & (h) \\
\hline & $D_{4 h}$ & & $\begin{array}{l}1.778(\mathrm{x} 4) \\
1.861(\mathrm{x} 2)\end{array}$ & & & (i (i)) \\
\hline & $O_{h}$ & & 1.792 & & & (i (ii)) \\
\hline & $O_{h}$ & & 1.846 & & & (i (iii)) \\
\hline & $D_{4 h}$ & ${ }^{3} \mathrm{~A}_{1 \mathrm{~g}}$ & $\begin{array}{l}1.850(\mathrm{x} 2) \\
1.849(\mathrm{x} 4)\end{array}$ & & & $(\mathrm{j}(\mathrm{i}))$ \\
\hline & $O_{h}$ & ${ }^{3} \mathrm{~A}_{1 \mathrm{~g}}$ & 1.911 & & & (j (ii)) \\
\hline & $D_{4 h}$ & & $1.916(\mathrm{x} 2)$ & & & (j (iii)) \\
\hline & $D_{3 d}$ & & $\begin{array}{l}1.908(\mathrm{x} 4) \\
1.910\end{array}$ & & & (i (iii)) \\
\hline & $D_{4 h}$ & ${ }^{3} \mathrm{~A}_{1 \mathrm{~g}}$ & & & $692\left(\mathrm{~A}_{2 \mathrm{u}}, 124\right), 671\left(\mathrm{E}_{\mathrm{u}}, 80\right), 608\left(\mathrm{~A}_{1 \mathrm{~g}}, 0\right), 580\left(\mathrm{~B}_{1 \mathrm{~g}}, 0\right), 569\left(\mathrm{~A}_{1 \mathrm{~g}}, 0\right)$ & (j (iv)) \\
\hline
\end{tabular}

${ }^{\dagger}$ The $D_{4 h}$ optimized structure is only $2 \mathrm{~kJ} \mathrm{~mol}^{-1}$ higher than the $D_{2 h}$ optimized structure for $\mathrm{PdF}_{4}$.

(a) this work BP86/ZORA-SO/TZ2P;

(b) this work B3LYP/aug-cc-pVTZ(F) aug-cc-pVTZ-PP(Pd) MCDHF RSC ECP;

(c) this work $\operatorname{CCSD}(\mathrm{T})$;

(d) $\mathrm{CISC} ;{ }^{1}$

(e) $\mathrm{MCPF}^{2}$

(f) B3LYP/6-311++G (F) SDD(Pd); ${ }^{3}$

(g) MCPF; ${ }^{4}$

(h) B3LYP/ECP(Pd) 6-311++G**(F); ${ }^{5}$

(i) (i) Non-relativistic LDA, (ii) Relativistic DHF, (iii) Relativistic Dirac-LDA; ${ }^{6}$

(j) (i) CCSD(T)/ATZ-PP, (ii) BLYP/ZORA/TZ2P, (iii) BLYP/ZORA-SO/TZ2P, (iv) B3LYP/AVZ-PP. ${ }^{7}$

The more recent work of Siddiqui ${ }^{8}$ is lacking in detail and rigour.

\section{References}

(1) Schwerdtfeger, P.; McFeaters, J. S.; Moore, J. J.; McPherson, D. M.; Cooney, R. P.; Bowmaker, G. A.; Dolg, M.; Andrae, D., Langmuir 1991, 7, 116-125.

(2) Siegbahn, P. E. M., Theor. Chim. Acta 1993, 86, 219-228.

(3) Cheng, L.; Wang, M. Y.; Wu, Z. J.; Su, Z. M., J. Comput. Chem. 2007, 28, 2190-2202.

(4) Siegbahn, P. E. M., Theor. Chim. Acta 1994, 87, 441-452. 
(5) Aullón, G.; Alvarez, S., Inorg. Chem. 2007, 46, 2700-2703.

(6) David, J.; Fuentealba, P.; Restrepo, A., Chem. Phys. Lett. 2008, 457, 42-44.

(7) Craciun, R.; Long, R. T.; Dixon, D. A.; Christe, K. O., J. Phys. Chem. A 2010, 114, 7571-7582.

(8) Siddiqui, S. A.; Bouarissa, N., Solid State Sci. 2013, 15, 60-65. 